\title{
Editorial
}

\section{Multirate Systems and Applications}

\author{
Yuan-Pei Lin, ${ }^{1}$ See-May Phoong, ${ }^{2}$ Ivan Selesnick, ${ }^{3}$ Soontorn Oraintara, ${ }^{4}$ and Gerald Schuller ${ }^{5}$ \\ ${ }^{1}$ Department of Electrical and Control Engineering, National Chiao-Tung University, Hsinchu 300, Taiwan \\ ${ }^{2}$ Department of Electrical Engineering and Graduate Institute of Communication Engineering, \\ National Taiwan University, Taipei 10617, Taiwan \\ ${ }^{3}$ Department of Electrical and Computer Engineering, Polytechnic University, Brooklyn, NY 11201, USA \\ ${ }^{4}$ Department of Electrical Engineering, The University of Texas at Arlington, Arlington, TX 76010, USA \\ ${ }^{5}$ Audio Coding for Special Applications Research Group, Fraunhofer Institute for Digital Media Technology (IDMT), \\ Langewiesener Strasse 22, 98693 Ilmenau, Germany
}

Received 24 January 2007; Accepted 24 January 2007

Copyright (c) 2007 Yuan-Pei Lin et al. This is an open access article distributed under the Creative Commons Attribution License, which permits unrestricted use, distribution, and reproduction in any medium, provided the original work is properly cited.

Filterbanks for the application of subband coding of speech were introduced in the 1970s. Since then, filterbanks and multirate systems have been studied extensively. There has been great success in applying multirate systems to many applications. Most notable of these applications include subband coding, signal analysis, and representation using wavelets, subband denoising, and so forth. Different applications also call for different filterbank designs and the topic of designing one-dimensional and multidimensional filterbanks for specific applications has been of great interest. Recently there has also been a lot of interest in applying multirate theories to the area of communication systems such as transmultiplexers, filterbank transceivers, and precoded systems. There are strikingly many dualities and similarities between multirate systems and multicarrier communication systems. Many problems in multicarrier transmission can be studied by extending results from multirate systems and filterbanks. This exciting research area is one that is of increasing importance.

The aim of this special issue is to bring forward recent developments on filterbanks and the ever expanding area of applications of multirate systems. In this special issue, there are a total of 13 papers, which are roughly grouped into 3 categories.

\section{THEORY, DESIGN, AND IMPLEMENTATION OF FILTERBANKS}

Yi Chen et al. developed two methods of designing quincunx filterbanks for image coding. Based on a lifting frame- work, a parameterization of quincunx filterbanks is employed to maximize coding gain subject to constraints on vanishing moments and frequency selectivity. The proposed methods are shown to be highly effective for image coding.

A frequency response masking approach to the design of cosine modulated $M$-channel filterbanks is developed by Linnéa et al. Using frequency response masking, this method can obtain a sharper prototype and hence analysis and synthesis filters with narrower transition bands. Furthermore, a lower complexity can be achieved at the cost of a slightly increased overall delay.

The problem of fixed wordsize implementation of lifting schemes is addressed by Tanja Karp. A reversible nonlinear discrete wavelet transform with a fixed wordsize based on lifting schemes is presented. It is shown that when the additions in the lifting steps are done using the modulus operation, overflows (if any) will cancel out. An analysis on the effect of finite wordsize implementation on the performance of image compression systems is performed. The results are useful for a practical implementation of lifting schemes.

The paper by M. Parfieniuk and A. Petrovsky proposes a new quaternionic lattice structures for four-channel paraunitary filterbanks. Quarternion multipliers are used as the paraunitary building blocks and they have the advantage that losslessness is preserved under coefficient quantization. The one-regularity condition can be expressed in terms of the lattice coefficients and can be satisfied even under finite precision. The proposed structure is useful for the design and implementation of four-channel paraunitary filterbanks.

A new characterization of real paraunitary two-channel filterbanks is proposed by M. Elena Domínguez Jiménez. The 
new formulation gives an explicit expression of all real FIR paraunitary filterbanks and it leads to a method that designs any two-channel paraunitary filterbanks directly, with no need of iteration procedures.

\section{APPLICATION OF FILTERBANK SYSTEMS TO COMMUNICATIONS}

Blind channel identification using redundant filterbank precoders is addressed by B. Su and P. P. Vaidyanathan. A generalized algorithm for solving the problem is proposed. The authors show how the parameters can be designed to jointly optimize the system performance and computational complexity. It is shown that the generalized algorithm outperforms the previous ones. In addition, a new concept of generalized signal richness and its properties are also investigated in the paper.

The issue of channel equalization in filterbank-based multicarrier systems is investigated by Tero Ihalainen et al. A new low-complexity per-subcarrier equalizer is proposed. A comprehensive performance analysis of the proposed system is presented and the performance of the proposed equalizer structures is compared to the cyclic-prefixed OFDM system, taking into account various practical issues like transmitter nonlinearity and frequency offsets. The study shows that the filterbank system is a promising candidate for multicarrier communications.

In a companion paper, Yuan Yang et al. investigate the use of exponentially modulated filterbanks for frequencydomain equalization in single-carrier systems. Two lowcomplexity equalizer structures are studied. It is demonstrated that the proposed filterbank-based single-carrier system outperforms the widely used DFT-based singlecarrier system, especially when there is narrowband interference.

The paper by Han-Ting Chiang et al. studies nonuniform filterbank transceivers for frequency selective channels. The authors propose a design method for jointly optimizing the frequency response and signal-to-interference ratio. Simulation results show that nonuniform filterbank transceivers with good frequency responses and high signalto-interference ratio can be obtained.

Frequency band reallocation is an important aspect of satellite-based communication systems. A variable oversampled complex modulated filterbank is introduced by H. Johansson and P. Löwenborg for flexible frequency band reallocation. Due to variable oversampling, the network is more flexible in accommodating various types of services. In addition, a lower complexity is simultaneously achieved due to inherent parallel processing.

\section{FILTERBANK SYSTEMS FOR SOUND AND ACOUSTICS APPLICATIONS}

In the paper by Arja Selin et al., filterbanks are applied to the recognition of bird sounds. Bird sounds can be tonal or in- harmonic, with the latter not easily captured by conventional spectral analysis methods. Using wavelet packet decomposition for feature extraction, inharmonic and transient sounds can be recognized with a high success rate.

Filterbanks have also been applied to crosstalk cancellation in spatial sound reproduction using multi-channel loudspeakers. The widespread use of the crosstalk cancellation system has been hampered by its heavy computational loading. The subband-based bandlimited cancellation system proposed by M. R. Bai and C.-C. Lee significantly reduces the complexity while having a performance comparable to that of the full-band system.

Convergence speed and complexity are known to be two important issues in acoustic echo cancellation associated with long echo paths. H. Choi and H.-D. Bae present a new subband affine projection method, combining subband filtering and affine projection, to address these two issues. The new algorithm outperforms both subband filtering and fullband affine projection methods in terms of convergence. At the same time, a lower complexity can be achieved.

\section{ACKNOWLEDGMENTS}

The editors would like to thank all the authors who submitted to this special issue and express their gratitude to all the reviewers for their valuable comments and suggestions. They also appreciate very much the support of EURASIP JASP Editorial Board. They hope that this special issue will stimulate more new developments and discoveries on the theories, designs, and applications of filterbank systems.

Yuan-Pei Lin

See-May Phoong

Ivan Selesnick

Soontorn Oraintara

Gerald Schuller

Yuan-Pei Lin was born in Taipei, Taiwan, 1970. She received the B.S. degree in control engineering from the National ChiaoTung University, Taiwan, in 1992, and the M.S. degree and the Ph.D. degree, both in electrical engineering from California Institute of Technology, in 1993 and 1997, respectively. She joined the Department of Electrical and Control Engineering of $\mathrm{Na}-$ tional Chiao-Tung University, Taiwan, in

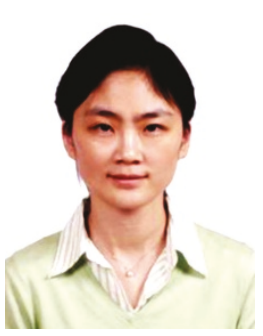
1997. Her research interests include digital signal processing, multirate filterbanks, and signal processing for digital communication, particularly in the area of multicarrier transmission. She is a recipient of $2004 \mathrm{Ta}$-You Wu Memorial Award. She served as an Associate Editor for IEEE Transaction on Signal Processing (2002-2006). She is currently an Associate Editor for IEEE Signal Processing Letters, IEEE Transaction on Circuits and Systems II, EURASIP Journal on Advances in Signal Processing, and Multidimensional Systems and Signal Processing, Academic Press. She is also a distinguished Lecturer of the IEEE Circuits and Systems Society for 2006-2007. 
See-May Phoong was born in Johor, Malaysia, in 1968. He received the B.S. degree in electrical engineering from the National Taiwan University (NTU), Taipei, Taiwan, in 1991 and the M.S. and Ph.D. degrees in electrical engineering from the California Institute of Technology (Caltech), Pasadena, Calif, USA, in 1992 and 1996, respectively. He was with the faculty of the Department of Electronic and Electrical

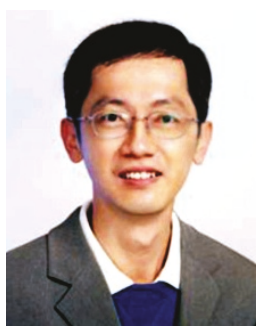
Engineering, Nanyang Technological University, Singapore, from September 1996 to September 1997. In September 1997, he joined the Graduate Institute of Communication Engineering and the Department of Electrical Engineering, NTU, as an Assistant Professor, and since August 2006, he has been a Professor. He is currently an Associate Editor for the IEEE Transactions on Circuits and Systems I. He has previously served as an Associate Editor for Transactions on Circuits and Systems II: Analog and Diginal Signal Processing (January 2002-December 2003) and IEEE Signal Processing Letters (March 2002-February 2005). His interests include multirate signal processing, filterbanks, and their application to communications. He received the Charles H. Wilts Prize (1997) for outstanding independent research in electrical engineering at Caltech. He was also a recipient of the Chinese Institute of Electrical Engineering's Outstanding Youth Electrical Engineer Award (2005).

Ivan Selesnick received the B.S., M.E.E., and Ph.D. degrees in electrical engineering in 1990, 1991, and 1996, respectively, from Rice University, Houston, Tex. In 1997, he was a Visiting Professor at the University of Erlangen-Nurnberg, Germany. He then joined the Department of Electrical and Computer Engineering, Polytechnic University, NY, USA, where he is an Associate Professor. His current research interests are

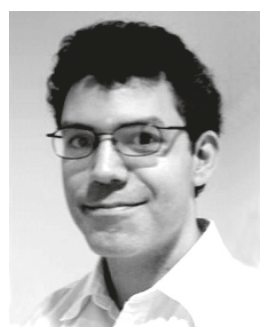
in the area of digital signal processing, wavelet-based signal processing, and non-Gaussian probability models. In 1991, he received a DARPA-NDSEG Fellowship. In 1996, Dr. Selesnick's Ph.D. dissertation received the Budd Award for Best Engineering Thesis at Rice University and an award from the Rice-TMC Chapter of Sigma Xi. He received an Alexander von Humboldt Award (1997) and a National Science Foundation Career Award (1999). He has been a Member of the IEEE Signal Processing Theory and Methods Technical Committee and he is an Associate Editor of the IEEE Transactions on Image Processing.

Soontorn Oraintara received the B.E. degree (with first-class honors) from King Monkut's Institute of Technology Ladkrabang, Bangkok, Thailand, in 1995, and the M.S. and Ph.D. degrees in electrical engineering from the University of Wisconsin, Madison, in 1996, and Boston University, Boston, Mass, USA, in 2000, respectively. He joined the Department of Electrical Engineering, University of Texas at Arlington

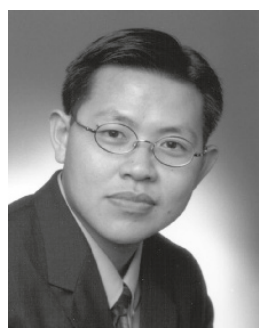
(UTA), as an Assistant Professor in July 2000, where he is currently an Associate Professor. From May 1998 to April 2000, he was an Intern and a Consultant with the Advanced Research and Development Group, Ericsson, Inc., Research Triangle Park, NC, USA, His current research interests are in the field of digital signal processing: wavelets, filterbanks, and multirate systems and their applications in data compression, image analysis, and biomedical signal processing. He is an Associate Editor for the IEEE Transactions on Signal Processing and the Circuits, Systems and Signal Processing Journal. He received the Technology Award from Boston University for his integer DCT invention (with Y. J. Chen and T. Q. Nguyen) in 1999. In 2003, he received the College of Engineering Outstanding Young Faculty Member Award from UTA. He represented Thailand in the International Mathematical Olympiad competitions and, respectively, received the Honorable Mention Award in Beijing, China, in 1990, and the bronze medal in Sigtuna, Sweden, in 1991.

Gerald Schuller is the head of the Audio Coding for Special Applications Research Group at the Fraunhofer Institute for Digital Media Technology in Ilmenau, Germany, since January 2002, and Adjunct Professor at the Technical University of Ilmenau. From spring of 2005 until spring of 2006, he was Deputy Professor for Applied Media Systems at that university. He received his Ph.D. degree from the University of

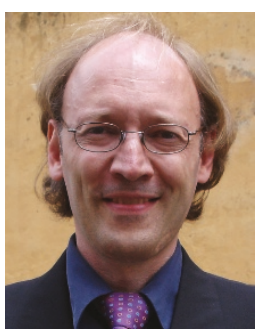
Hanover in 1997. From 1998 to 2001, he was a member of technical staff at Bell Laboratories, Lucent Technologies, and Agere Systems, a Lucent spin-off. There he worked in the Multimedia Communications Research Laboratory. He was an Associate Editor of the IEEE Transactions on Speech and Audio Processing from 2002 until 2006, and is an Associate Editor of the IEEE Transactions on Signal Processing since 2006. He is a Member of the IEEE Technical Committees on Audio and Electroacoustics, on Speech and Language Processing, and Member of the Audio Engineering Society (AES) Technical Committees on Coding of Audio Signals, and on Signal Processing. 